DOI: $10.3724 /$ SP.J.1218.2013.00686

\title{
基于刚度模糊观测的假手反演控制策略
}

\author{
吴常铖，宋爱国，章华涛，冯超 \\ (东南大学仪器科学与工程学院, 江苏 南京 210096)
}

\begin{abstract}
摘 要: 为了使假手能够完成对不同刚性物体的柔顺抓取, 提出了一种带有刚度模糊观测的反演控制策略, 以 满足假手在自由空间内的速度比例控制和在约束空间内的柔性握力控制，两个控制过程采用同一个反演控制器实 现. 设计了轨迹规划器, 对假手的期望开合速度、握力进行规划, 将速度控制和握力控制转换为位置控制; 设计了基 于位置的反演控制器, 并采用李亚普诺夫稳定性理论证明了该反演控制器的稳定性; 设计了刚度模糊观测器, 实时 调整假手模型参数, 均衡被抓握物体对假手控制策略的影响, 并实现假手在自由空间和约束空间控制过程的过渡. 对所提出的控制策略进行了实验验证，实验结果证明了该控制策略的有效性.
\end{abstract}

关键词: 假手; 模糊观测; 反演控制

中图分类号: TP911.7, TP241

文献标识码: A

文章编号: 1002-0446(2013)-06-0686-06

\section{A Backstepping Control Strategy for Prosthetic Hand Based on Fuzzy Observation of Stiffness}

\author{
WU Changcheng, SONG Aiguo, ZHANG Huatao, FENG Chao
}

(School of Instrument Science and Engineering, Southeast University, Nanjing 210096, China)

\begin{abstract}
In order to make the prosthetic hand complete compliant grasp of different rigid objects, a prosthetic hand control strategy based on fuzzy observation of stiffness is proposed to meet the needs of the speed ratio control in free space and the grip force flexible control in constraint space. Both control process are realized by the same backstepping controller. The expected opening and closing speed, and grip strength are planned by the designed trajectory planner. The conversion from the speed control and the grip force control to the position control is realized. The position backstepping controller is designed and its stability is proved based on Lyapunov stability theory. The designed fuzzy stiffness observer adjusts the parameters of the model in real time, balances the influence of the grasped objects on the control strategy, and switches the control process between free space and constraint space. The control strategy is verified by experiments, and the effectiveness of the control strategy is proved.
\end{abstract}

Keywords: prosthetic hand; fuzzy observation; backstepping control

\section{1 引言 (Introduction)}

假手是辅助手臂截肢患者完成力所能及工作的 重要工具, 如何使得假手能像自然人手一样随心所 欲地抓握物体，是假手研究的前进方向. 假手的控 制经历了开关控制、肌电阈值控制、肌电比例控制 等几个阶段, 肌电比例控制假手由于其动作自然、 与人体操作习惯相似而受到广泛关注. 假手开合速 度的比例控制和握力的柔性控制是假手研究的重 点.

针对机械手的握力控制, 文 [1] 采用切换理论, 根据机械手上压力传感器测得的压力值, 将机械手 的抓取动作分为有压力和无压力两个阶段进行, 实
现机械手柔性抓取. 文 [2] 将反演控制器与滑模控 制器和握力反馈控制器相结合构建反演滑模混合 控制策略, 并通过对比实验证明了控制策略的有效 性. 文 [3] 提出在手指指尖和手指关节处定义虚拟 弹簧阻尼器的方法来实现假手握力的精确控制. 为 使假手能够完成多种精细动作, 文 [4] 设计了多自 由度假手 HIT/DLR, 文 [5] 提出在自由空间和约束 空间中分别使用基于位置的阻抗控制和力跟踪控 制, 两个控制模式采用同一个阻抗控制器实现, 通 过模糊观测器进行控制模式切换, 提出了自适应滑 模摩擦力补偿方法, 使得系统跟踪误差在有限时间 内收玫, 并在文 [4] 设计的 HIT 假手上进行实验,

基金项目：国家自然科学基金资助项目（61272379，61325018）；江苏省自然科学基金重点资助项目（BK2010063）; 江苏省产学研前瞻性项 目 (BY2012201).

通讯作者: 宋爱国, a.g.song@seu.edu.cn收稿/录用/修回: 2013-01-24/2013-03-25/2013-06-24 
证明了控制策略的有效性. 文 [6] 通过神经模糊推 理得到假手反向运动学模型, 通过回归矩阵得到动 力学模型, 采用混合自适应控制策略减小干扰和位 置参数的影响, 仿真结果表明, 与 PID 控制相比, 该 方法具有较好的握力跟踪效果. 考虑接触环境的不 同, 文 [7] 采用模糊控制算法, 定义屈从性和正切力 比率作为模糊控制器的输入, 模糊控制器输出假手 手指闭合的调整量, 从而实现假手握力的控制, 文 中定义的正切力比率由于需要借助于安装在假手手 腕部位的腕力传感器来测量被抓握物体的重量, 因 而在使用过程中假手的移动或抓握物体角度的不同 都会给控制精度带来影响. 文 [8] 通过对环境刚度 进行模糊自适应估计、修改多模型模糊力控制器的 加权系数来实现机器人在未知环境中精确的接触力 控制, 并通过仿真证明了控制器的可行性, 该方法 虽然考虑到环境的影响, 但是需要预先针对几种典 型的接触环境设计相应的模糊控制器, 为提高控制 精度, 系统应预先设计尽可能多的模糊控制器, 计 算量较大. 上述控制方法对于假手握力的精确控制 都具有一定的有效性, 但大多数没有考虑被抓握物 体刚度对控制策略的影响, 且在通用性及商业化假 手的应用上存在一定的局限性.

针对上述问题, 为了使假手在抓握不同刚度物 体时能够精确地跟踪肌电信号所表现出的期望握 力, 提高假手抓握不同刚度的柔性物体的成功率, 本文提出了一种带有刚度模糊观测器的反演控制策 略, 通过刚度模糊观测器调节假手模型参数, 均衡 被抓握物体对控制策略的影响, 设计一个基于位置 的反演控制器实现假手在自由空间内的速度比例控 制和在约束空间内的握力跟踪控制.

\section{2 控制器设计 (Design of the controller)}

本文设计的假手控制策略如图 1 所示, 实现自 由空间内的速度比例控制和约束空间内的握力跟踪 控制, 两个控制过程采用同一个基于位置的反演控 制器实现, 并通过刚度观测器进行切换. $F_{\mathrm{d}}$ 为肌电 传感器测得的人体手臂肌电信号, 自由空间内 $F_{\mathrm{d}}$ 对 应于假手的期望开合速度, 约束空间内 $F_{\mathrm{d}}$ 对应于假 手的期望握力. 轨迹规划器将假手的期望开合速度、 期望握力映射为期望角度 $\theta_{\mathrm{d}}$, 从而将速度控制和握 力控制转换为位置控制. 刚度模糊观测器实时修正 假手模型中的阻尼项, 均衡不同刚度被抓握物体对 控制策略的影响. $F_{\mathrm{n}}$ 为假手的实际握力, 即被抓握 物体和假手手指间的压力, $x_{1}$ 为当前假手手指的位 置, 即假手上直流电机的转动角度, $x_{2}$ 为假手上直 流电机的转动角速度, $K_{\mathrm{n}}$ 为比例因子, $K_{\mathrm{nd}}$ 为微分比
例因子, $K_{\mathrm{p}}$ 为转换因子, $u$ 为施加在假手电机两端 的电压, $k$ 为刚度观测器输出的被抓握物体的刚度.

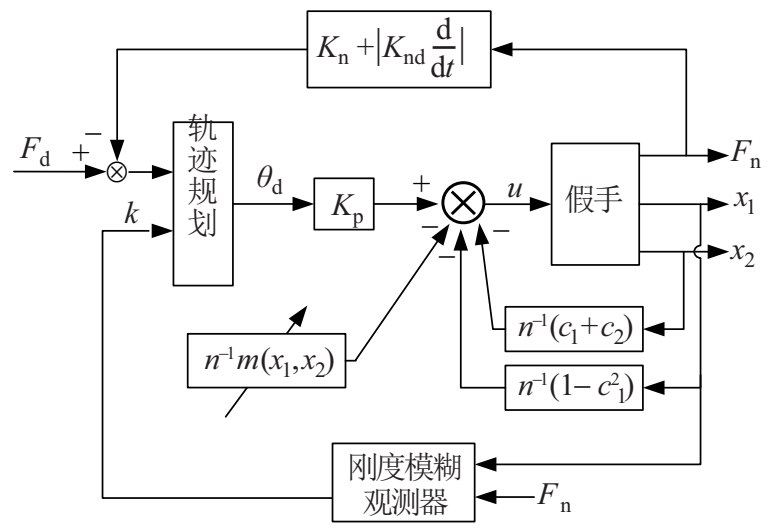

图 1 控制策略框图

Fig.1 Block diagram of the control strategy

自由空间内, 轨迹规划器输出为

$$
\theta_{\mathrm{d}}=\theta_{0}+\int\left(F_{\mathrm{d}}-K_{\mathrm{n}} F_{\mathrm{n}}-K_{\mathrm{nd}} \frac{\mathrm{d}}{\mathrm{d} t} F_{\mathrm{n}}\right) \mathrm{d} t=\theta_{0}+\int F_{\mathrm{d}} \mathrm{d} t
$$

其中, $\theta_{0}$ 为假手从约束空间切换至自由空间时手指 所在的位置.

约束空间内, 假手握力大小与电机转动角度之 间的关系受被抓物体的刚度和假手机械结构变形等 因素的影响, 由于设计的假手握力范围较小 (在 0 $\sim 30 \mathrm{~N}$ 之间), 在忽略假手机械结构变形影响的情 况下, 约束空间内轨迹规划器的输出为

$$
\theta_{\mathrm{d}}=\theta_{\mathrm{n}}+\frac{F_{\mathrm{d}}-\left(K_{\mathrm{n}} F_{\mathrm{n}}+K_{\mathrm{nd}} \frac{\mathrm{d}}{\mathrm{d} t} F_{\mathrm{n}}\right)}{k}
$$

其中, $\theta_{\mathrm{n}}$ 为当前假手手指所在的位置.

\section{1 反演控制器设计}

反演控制法由 Kokotovic 等人于 1991 年首次提 出, 用于处理线性系统和某些非线性系统, 通常与 李亚普诺夫型自适应律结合使用, 在改善过渡过程 方面表现出了较好的特性, 适用于可以实现状态线 性化或具有严格参数反馈的不确定非线性系统 ${ }^{[9]}$.

选取假手的数学模型为

$$
\left\{\begin{array}{l}
\dot{x}_{1}=x_{2} \\
\dot{x}_{2}=m\left(x_{1}, x_{2}\right)+n u \\
m\left(x_{1}, x_{2}\right)=-\frac{B}{J} x_{2}-\frac{D}{J}
\end{array}\right.
$$

其中, $x_{1}$ 为电机转动的角位移, $x_{2}$ 为电机转动的角 速度, $B 、 J 、 D$ 分别代表系统的阻尼、惯性和未知非 线性阻尼, $u$ 为控制器输出控制量, 即电机的控制电 压. 
利用虚拟反馈定义两个误差子系统:

$$
\left\{\begin{array}{l}
Z_{1}=x_{1} \\
Z_{2}=x_{2}-\alpha_{1}\left(x_{1}\right)
\end{array}\right.
$$

其中 $\alpha_{1}\left(x_{1}\right)$ 为虚拟控制量, 是 $x_{2}$ 的估计值.

$$
\dot{Z}_{1}=\dot{x}_{1}=x_{2}
$$

取 $\alpha_{1}\left(x_{1}\right)=-c_{1} Z_{1}, c_{1}>0$, 则:

$$
\dot{Z}_{1}=Z_{2}+\alpha_{1}\left(x_{1}\right)=Z_{2}-c_{1} Z_{1}
$$

定义第 1 个误差子系统的李亚普诺夫函数为

$$
\begin{aligned}
V_{1} & =\frac{1}{2} Z_{1}^{2} \\
\dot{V}_{1} & =Z_{1} \dot{Z}_{1}=-c_{1} Z_{1}^{2}+Z_{1} Z_{2} \\
\dot{Z}_{2} & =\dot{x}_{2}-\dot{\alpha}_{1}\left(x_{1}\right) \\
& =m\left(x_{1}, x_{2}\right)+n u+c_{1} \dot{Z}_{1} \\
& =m\left(x_{1}, x_{2}\right)+n u-c_{1}^{2} Z_{1}+c_{1} Z_{2}
\end{aligned}
$$

定义第 2 个误差子系统的李亚普诺夫函数为

$$
\begin{aligned}
V_{2}= & V_{1}+\frac{1}{2} Z_{2}^{2} \\
\dot{V}_{2}= & \dot{V}_{1}+Z_{2} \dot{Z}_{2} \\
= & -c_{1} Z_{1}^{2}+Z_{1} Z_{2}+Z_{2}\left[m\left(x_{1}, x_{2}\right)+n u+c_{1} \dot{Z}_{1}\right] \\
= & -c_{1} Z_{1}^{2}+Z_{1} Z_{2}+ \\
& Z_{2}\left[m\left(x_{1}, x_{2}\right)+n u-c_{1}^{2} Z_{1}+c_{1} Z_{2}\right]
\end{aligned}
$$

取电机两端电压 $u$ 的控制律为

$$
u=\frac{1}{n}\left[-m\left(x_{1}, x_{2}\right)-\left(1-c_{1}^{2}\right) Z_{1}-\left(c_{2}+c_{1}\right) Z_{2}\right]
$$

将式 (12) 代入式 (11), 则:

$$
\dot{V}_{2}=-c_{1} Z_{1}^{2}-c_{2} Z_{2}^{2} \leqslant 0, \quad c_{1}>0, c_{2}>0
$$

由李亚普诺夫稳定性理论可知设计的控制系统 可在有限时间内达到稳定状态, 具有稳定性. 通过 调节式 (12) 中 $c_{1} 、 c_{2}$ 可以调节控制器的响应特性.

\section{2 刚度模糊观测器设计}

为均衡不同刚度的被抓握物体对假手控制策略 的影响, 文章通过设计刚度模糊观测器对 $m\left(x_{1}, x_{2}\right)$ 进行调整，以实现对非刚性物体的柔性抓取.

定义被抓握物体的刚度 $k\left(\mathrm{~N} /\left(^{\circ}\right)\right)$ :

$$
k=\frac{F_{\mathrm{n}}}{x_{0}-x_{1}}
$$

其中, $x_{0}$ 为被抓握物体的初始尺寸, 即假手手指与 被抓握物体初次接触时假手手指的位置.
表 1 模糊推理规则

Tab.1 Fuzzy reasoning rules

\begin{tabular}{c|c|cccc}
\hline \multirow{2}{*}{\multicolumn{2}{c|}{$w$}} & \multicolumn{4}{|c}{$k$} \\
\cline { 3 - 6 } \multicolumn{2}{c}{} & ZO & S & M & L \\
\hline \multirow{4}{*}{$\dot{k}$} & NL & ZO & ZO & S & M \\
& NS & ZO & S & S & M \\
& ZO & ZO & S & M & L \\
& PS & S & S & M & L \\
& PL & S & M & L & L \\
\hline
\end{tabular}

设计的模糊观测器以被抓握物体的刚度 $k$ 及其 变化率 $\dot{k}$ 作为输入, 模糊观测器输出 $B$, 采用三角 和梯形隶属函数, $k 、 \dot{k}$ 和 $B$ 的隶属函数分别如图 2、 图 3、图 4 所示. $K$ 定义 4 个模糊子集 $\{\mathrm{ZO}, \mathrm{S}, \mathrm{M}$, $\mathrm{L}\}, \dot{k}$ 定义 4 个模糊子集 $\{\mathrm{NL}, \mathrm{NS}, \mathrm{ZO}, \mathrm{PS}, \mathrm{PL}\}, B$ 定义 4 个模糊子集 $\{\mathrm{ZO}, \mathrm{S}, \mathrm{M}, \mathrm{L}\}$. 模糊推理规则 见表 1 , 图 5 为模糊观测器的输入输出关系图.

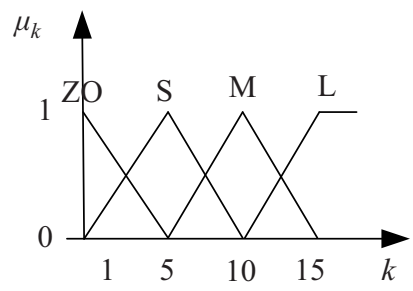

图 $2 k$ 的隶属函数

Fig.2 Membership function of $k$

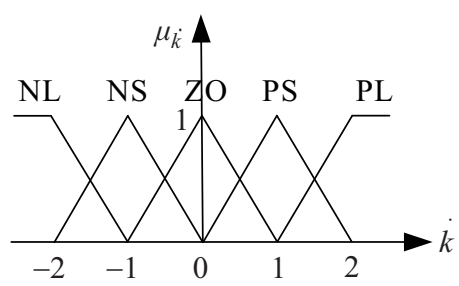

图 $3 \dot{k}$ 的隶属函数

Fig.3 Membership function of $\dot{k}$

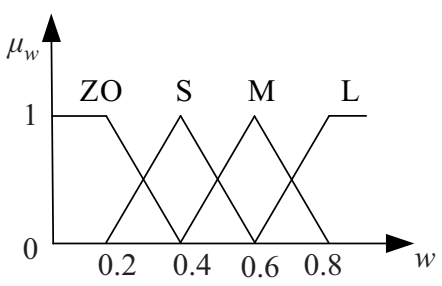

图 $4 w$ 隶属函数

Fig.4 Membership function of $w$

通过刚度模糊观测器的输出对假手模型中的 $m\left(x_{1}, x_{2}\right)$ 进行调整以得到期望的控制效果, 调整方 法如下:

$$
m\left(x_{1}, x_{2}\right)=-(1+w) \frac{B}{J} x_{2}-\frac{D}{J}
$$




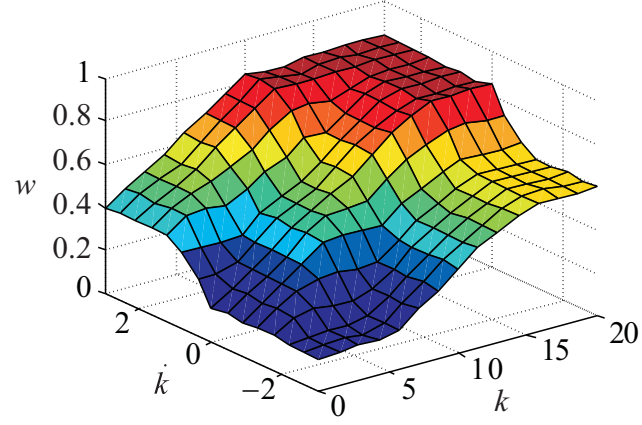

图 5 输入输出关系表面图

Fig.5 Surface map of input-output relationship

\section{3 实验及分析（Experiments and analyses）}

\section{1 实验装置}

实验对象为东南大学机器人传感与控制技术实 验室研制的 MPH-II 型肌电假手, 佩戴上硅胶手套后 与人手外形一样, 为单自由度双动作假手, 由一个 直流电机控制假手的张开、闭合, 假手上的光电编 码盘用于检测假手的张开角度，指尖位置安装的触 点力传感器用于检测假手的捏握力, 如图 6 所示.

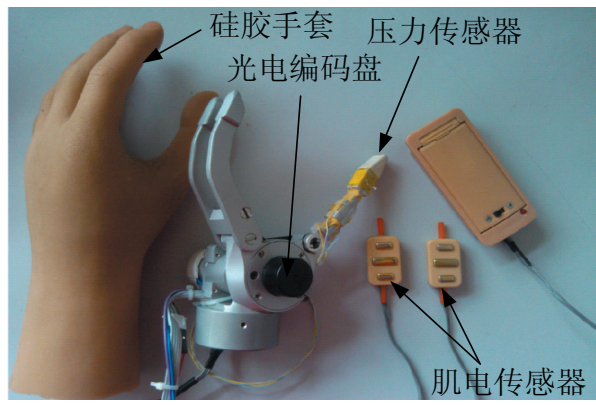

图 6 MPH-II 肌电假手

Fig.6 MPH-II EMG prosthetic hand

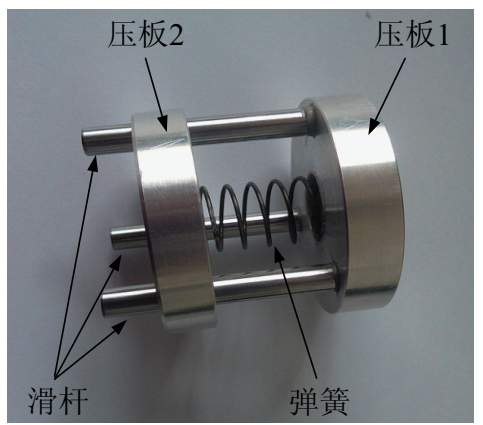

图 7 被抓握对象

Fig.7 Grasped object

MPH-II 采用 C8051F320 单片机作为假手控制 系统的处理核心, 采用 L298 对 PWM 电机驱动信号 进行功率放大, 系统中信号的采样速率为 $2 \mathrm{kHz}$.

约束空间内进行力跟踪实验采用如图 7 所示的 被抓握物体, 由两块压板、弹簧和限制压板运动方 向的滑竿组成, 弹簧与两块压板相连, 滑竿的一端
与压板 1 相连，压板 2 上的通孔穿过滑竿的另一端, 通过更换弹簧可以调节被抓握对象的刚度.

\section{2 自由空间的速度位置跟踪实验}

假手处于闭合状态，进行速度位置跟踪实验， 输入期望开合速度, 经轨迹规划器后输出期望位置, 实验结果如图 8～图 11 所示. 实验结果表明, 设计 的假手控制策略在自由空间内能够快速准确地跟踪 期望的开合速度.

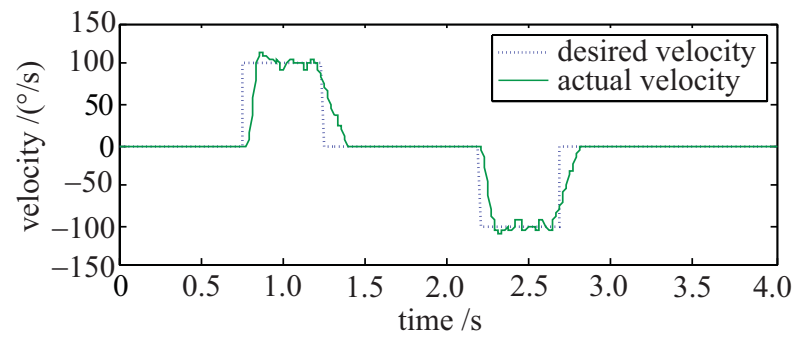

图 8 速度跟踪曲线

Fig.8 Velocity tracking curve

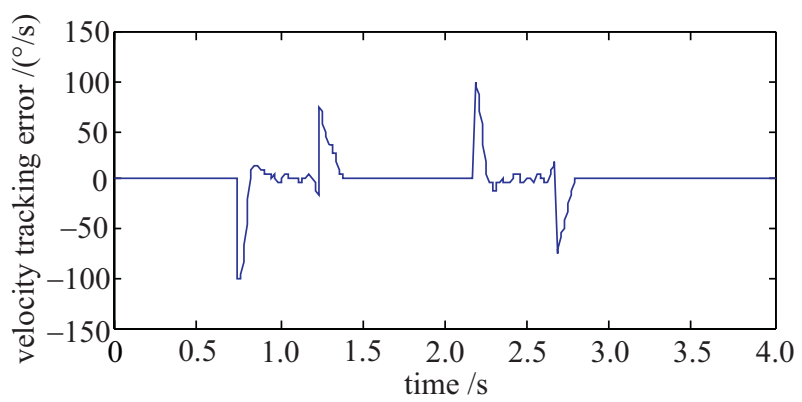

图 9 速度跟踪误差曲线

Fig.9 Velocity tracking error curve

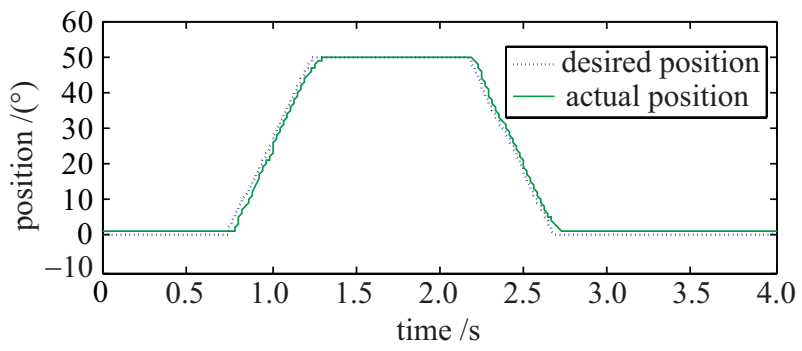

图 10 位置跟踪曲线

Fig.10 Position tracking curve

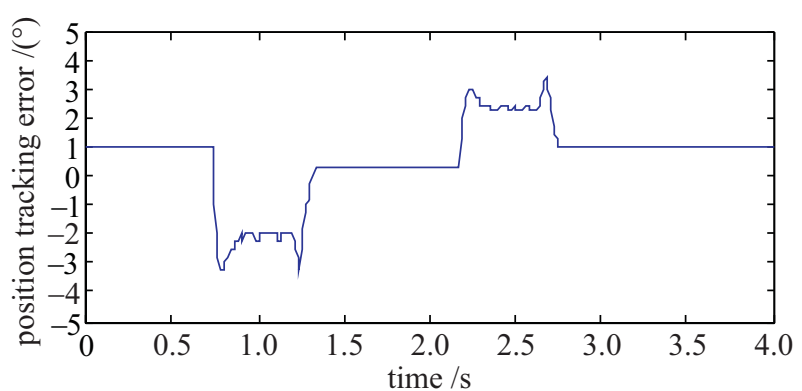

图 11 位置跟踪误差曲线

Fig.11 Position tracking error curve 


\section{3 握力跟踪实验}

让假手手指与物体接触, 进行握力跟踪实验, 如图 12 所示. 阶跃变化握力跟踪和线性变化握力跟 踪的实验结果分别如图 13、图 14 所示, 实验结果表 明, 设计的假手控制策略在约束空间内能有效地跟 踪期望握力的变化.

更换图 7 所示装置中的弹簧, 进行图 13 和图 14 所示的阶跃变化握力和线性变化握力跟踪实验, 实 验结果如表 2 所示, 表中给出了握力跟踪误差绝对 值均值 $\bar{e}$ 和 $\bar{e}$ 的均方根 $R$ 的实验数据.

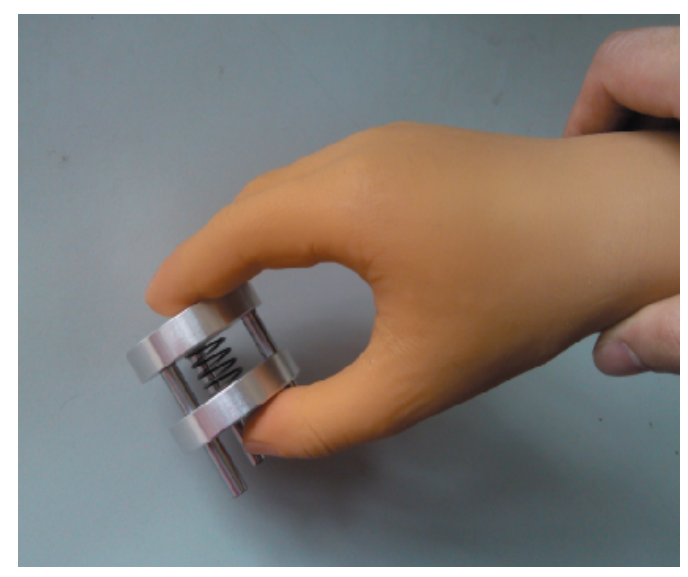

图 12 握力跟踪实验

Fig.12 Grip force tracking experiment

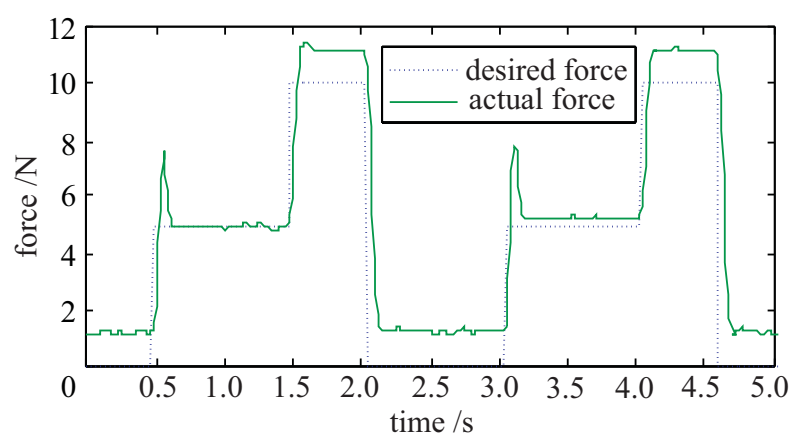

(a) 阶跃握力跟踪曲线

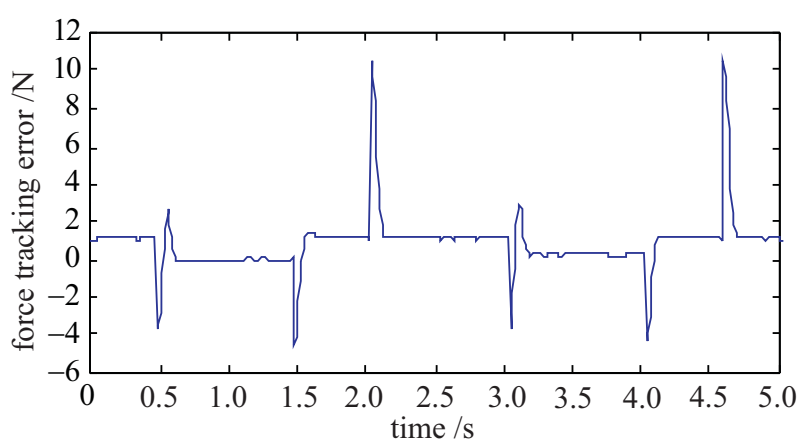

(b) 阶跃握力跟踪误差曲线

图 13 阶跃握力跟踪实验结果

Fig.13 Result of step grip force tracking experiment

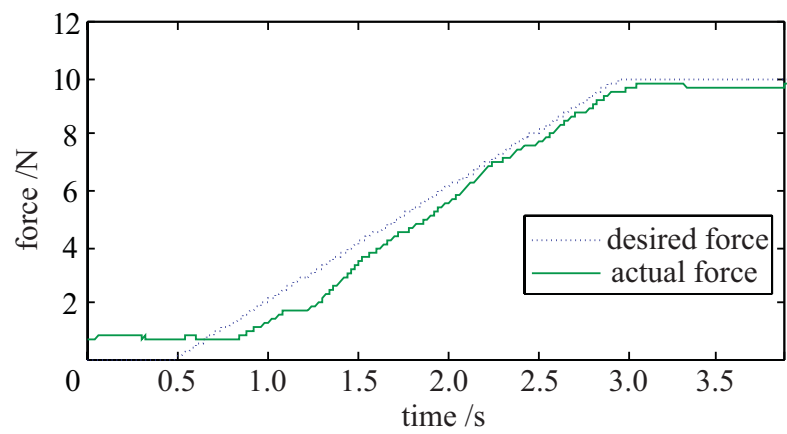

(a) 线性握力跟踪曲线

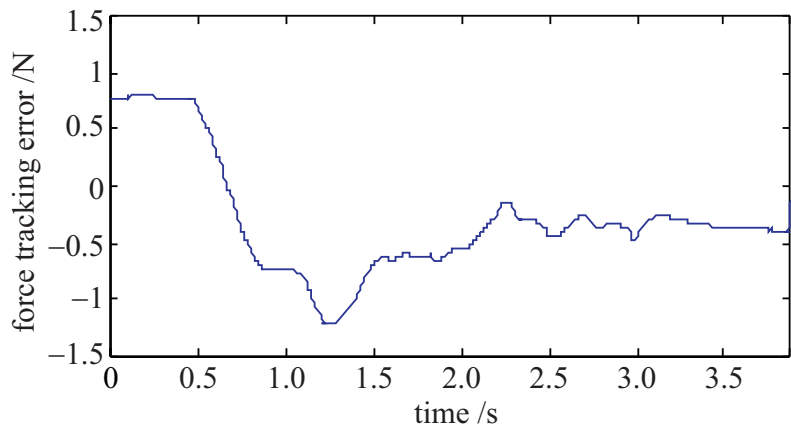

(b) 线性握力跟踪误差曲线

图 14 线性握力跟踪实验结果

Fig.14 Result of linear grip force tracking experiment

表 2 不同刚度物体的抓握实验结果

Tab.2 Grasp results of different objects

\begin{tabular}{cccccc}
\hline \multirow{2}{*}{ 编号 } & $\begin{array}{c}\text { 弹簧 } \\
\text { 弹性系数 }\end{array}$ & \multicolumn{2}{l}{ 阶跃力跟踪实验 } & \multicolumn{2}{c}{ 线性力跟踪实验 } \\
\cline { 3 - 7 } & $/(\mathrm{N} / \mathrm{mm})$ & $\mathrm{N}$ & $\mathrm{N}$ & $\overline{\mathrm{N}}$ & $R$ \\
\hline 1 & 0.5 & 1.76 & 1.56 & 0.82 & 1.00 \\
2 & 1 & 1.85 & 1.83 & 1.46 & 1.33 \\
3 & 2 & 1.98 & 2.06 & 0.88 & 1.02 \\
4 & 4 & 2.21 & 1.86 & 1.43 & 1.27 \\
5 & 10 & 1.87 & 1.96 & 1.22 & 1.10 \\
\hline
\end{tabular}

\section{4 结论 (Conclusion)}

本文设计的假手控制策略采用同一个反演控制 器实现了假手在自由空间内的速度比例控制和约束 空间内的握力跟踪控制, 两个控制过程通过刚度模 糊观测器进行切换. 设计的轨迹规划器对假手自由 空间和约束空间的运动进行规划. 利用李亚普诺夫 稳定性理论设计了基于位置的反演控制器. 设计的 刚度模糊观测器能够有效地均衡不同刚度被抓握物 体对控制策略的影响, 提高了假手抓握物体的柔顺 性.

\section{参考文献（References）}

[1] 李玉民. 机械手柔性抓取切换控制系统设计与仿真分析 [J]. 国外电子测量技术, 2010，29(10): 30-33. 
Li Y M. Switching control design and simulation analysis of the flexible control system of the manipulator[J]. Foreign Electronic Measurement Technology, 2010, 29(10): 30-33.

[2] Engeberg E D, Meek S G. Backstepping and sliding mode control hybridized for a prosthetic hand[J]. IEEE Transactions on Neural Systems and Rehabilitation Engineering, 2009, 17(1): 70-79.

[3] Fassih A, Naidu D S, Chiu S, et al. Precision grasping of a prosthetic hand based on virtual spring damper hypothesis[C]//2010 5th Cairo International Bio-medical Engineering Conference. Piscataway, USA: IEEE, 2010: 79-82.

[4] Huang H, Jiang L, Zhao D W, et al. The development on a new biomechatronic prosthetic hand based on under-actuated mechanism[C]//EEE/RSJ International Conference on Intelligent Robots and Systems. Piscataway, USA: IEEE, 2006: 37913796.

[5] 张庭, 姜力, 刘宏. 仿生假手抓握力控制策略 [J]. 机器 人, 2012, 34(2): 190-196.

Zhang T, Jiang L, Liu H. A grasping force control strategy for anthropomorphic prosthetic hand[J]. Robot, 2012, 34(2): 190196.

[6] Chen C H, Naidu D S, Perez-Gracia A, et al. A hybrid adaptive control strategy for a smart prosthetic hand[C]//31st Annual International Conference of the IEEE Engineering in Medicine and Biology Society: Engineering the Future of Biomedicine. Piscataway, USA: IEEE, 2009: 5056-5059.

[7] 罗志增, 杨广映. 基于触觉和肌电信号的假手模糊控制方 法研究 [J]. 机器人, 2006, 28(2): 224-228.

Luo Z Z, Yang G Y. Prosthetic hand fuzzy control based on touch and myoelectric signal[J]. Robot, 2006, 28(2): 224-228.

[8] 李战明, 李二超, 李炜, 等. 基于环境刚度模糊自适应估计 的机器人力控制 [J]. 工业仪表与自动化装置, 2010(4): 110112.

Li Z M, Li E C, Li W, et al. The robotic force control based on fuzzy adaptive estimation of environmental stiffness[J]. Industrial Instrumentation \& Automation, 2010(4): 110-112.

[9] 乔继红. 反演控制方法与实现 [M]. 北京: 机械工业出版 社, 2012.

Qiao J H. Backstepping control methods and implementation[M]. Beijing: China Machine Press, 2012.

\section{作者简介:}

吴常铖 (1987-), 男, 博士生. 研究领域: 康复医疗仪器, 机器人传感与控制技术.

宋爱国 (1968-), 男, 博士, 教授, 博士生导师, 研究领 域: 机器人传感与控制技术, 信号处理, 遥操作技 术等. 